\title{
Development of a Model Running Facility for a Study on under-floor Flow ${ }^{*}$
}

\author{
Atsushi IDO ${ }^{* *}$ and Shuya YOSHIOKA ${ }^{* * *}$ \\ ${ }^{* *}$ Railway Technical Research Institute, \\ 2-8-38 Hikari-cho Kokubunji-shi, 185-8540 Tokyo, Japan \\ E-mail: ido@rtri.or.jp \\ ${ }^{* * *}$ Ritsumeikan University, \\ 1-1-1 Noji Higashi, Kusatsu, Shiga 525-8577 Japan
}

\begin{abstract}
We developed a model running facility to study the flow of the under-floor of railway car. The facility was set up in the test section of the Towing Wind Tunnel Facility where by a hood we can eliminate disturbances, such as natural wind. We carried out the model running tests and measured the velocity distribution under the model car. The velocity distribution was almost the same as in the rear cars from the car No.3, and the velocity between the rails was less than the velocity outside of the rail.
\end{abstract}

Key words: Train, Model Running Facility, Under-floor Flow, Towing Wind Tunnel Facility

\section{Introduction}

The air flow between the underside of a car and a track is one of main causes of ballast flying phenomena ${ }^{1)}$, snow accretion ${ }^{2)}$, aerodynamic $\operatorname{drag}^{3)}$, aerodynamic noise ${ }^{4)}$ and so on. To solve these problems due to the induced flow by the train, it is necessary to study the under-floor airflow.

We have some means available for studying under-floor flow, such as on-track tests, wind tunnel tests, CFD and others. Considering cost performance, the wind tunnel tests are suitable for studying the subject. Previously, we studied this issue using a large-scale wind tunnel, equipped with a boundary layer suction and a moving belt ${ }^{5)}$ to reproduce under-floor flow. However, the wind tunnel test has the following problems:

(1) It is difficult to estimate the velocity of the airflow above the track while the train is passing by, because we cannot install a sensor on the moving belt.

(2) Since the train makeup is long, we set only one or two cars on the belt, and could not estimate the influence of the train length.

Therefore, we have developed a model running facility to solve these problems. We report the development of a model running facility and the results of measurements of the under-floor flow by this facility. 


\section{Model running facility}

\subsection{Towing wind tunnel facility}

It is essential to remove disturbances, such as natural wind, to estimate the flow velocity near the ground by means of the model running facility. Therefore, we have better install the facility indoors. Moreover, to run the models at sufficient speed requires a large space in terms of both length and cross section. Therefore, we installed the facility in the "Towing Wind Tunnel Facility") of Tohoku University shown in Fig.1, which has a large test-section. The Towing Wind Tunnel Facility was constructed on a former MAGLEV testing track (guideway) owned by Railway Technical Research Institute. The testing track of the Towing Wind Tunnel Facility is constructed in the first $1900 \mathrm{~m}$ of the guideway of $6900 \mathrm{~m}$ in total. It has the closed section by a hood and shutters that is $515 \mathrm{~m}$ long and we call it test-section, as shown in Fig. 2. The test section, is $515 \mathrm{~m}$ long, $3.46 \mathrm{~m}$ wide, and has a maximum height of $3.325 \mathrm{~m}$. We carried out model running tests there.

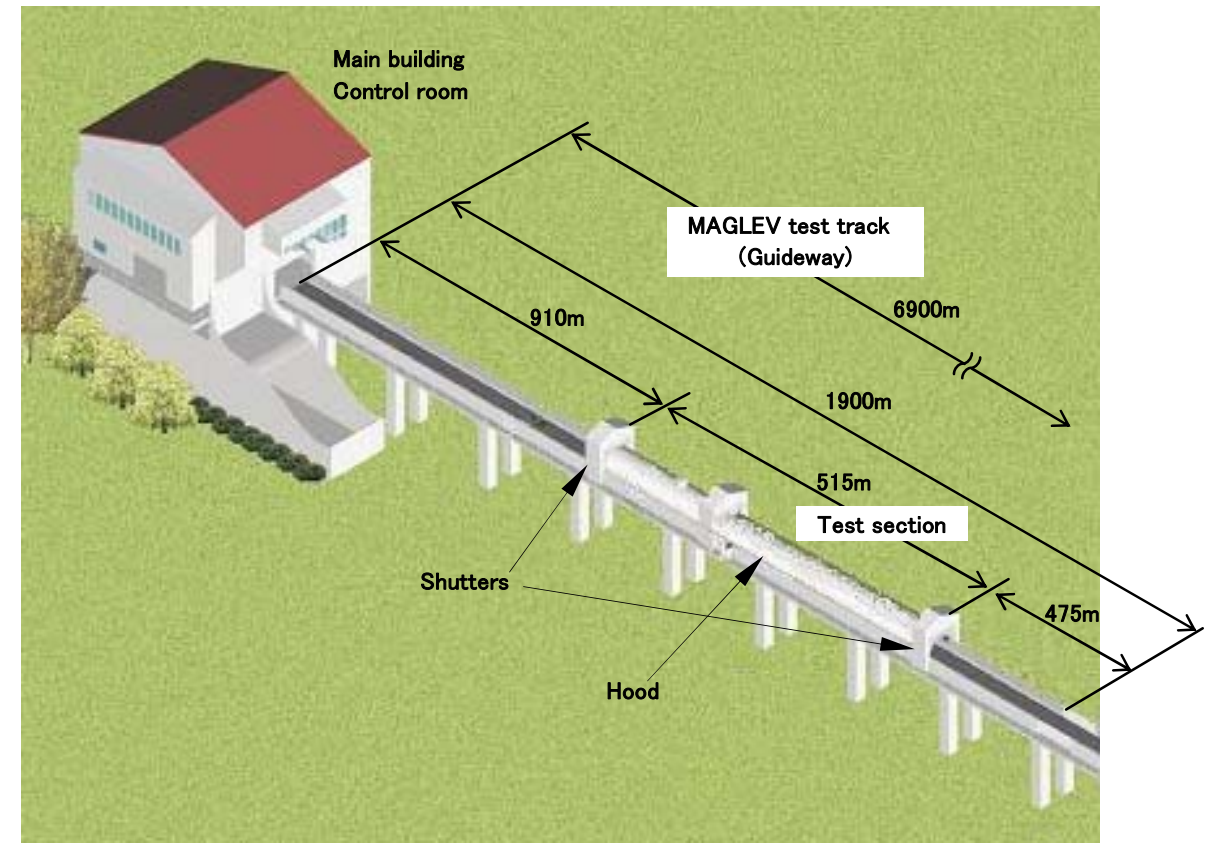

Fig. 1 Towing wind tunnel facility 

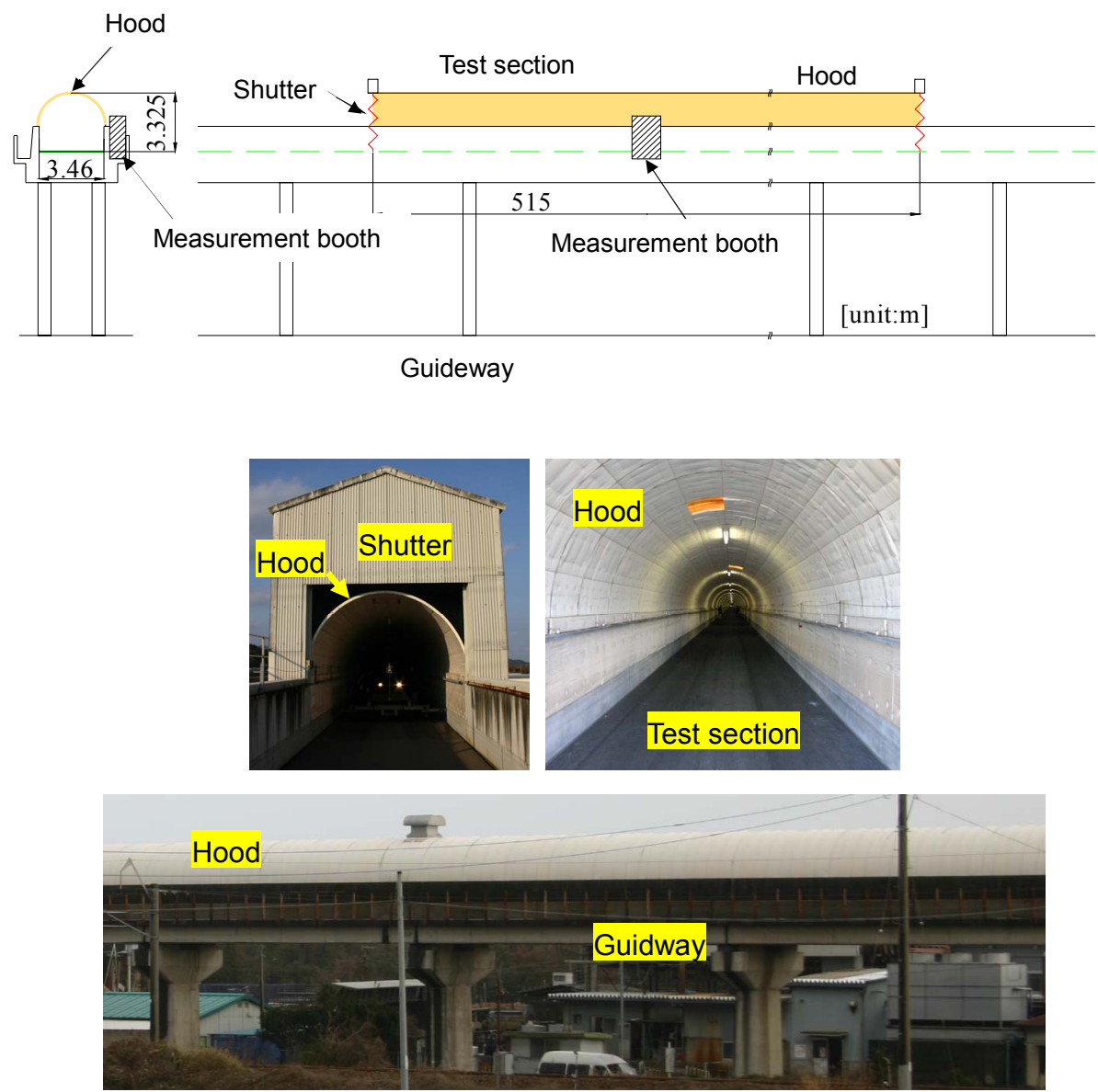

Fig. 2 Test section of Towing Wind Tunnel Facility

\subsection{Temporary track panel}

The surface of the ground in the Towing Wind Tunnel Facility is paved with asphalt and flat. To run the model train in our experiments, we laid the rails that are $460 \mathrm{~m}$ long in the test section of the Towing Wind Tunnel Facility.

We adopted a commercial rail to reduce the costs of long rails. Their scale is $1 / 8.4$. A track panel, shown in Fig. 3, consisting of rails and sleepers is commercially available. The pitch of the sleepers is $75 \mathrm{~mm}$ - they are $3.075 \mathrm{~m}$ long and weigh $12 \mathrm{~kg}$. We obtained the 150 track panels, and constructed the $450 \mathrm{~m}$ of the rails. The track panels were set up at the centre of the direction of the width of the ground. The track panels constructed temporarily are maintained by friction by their weight.

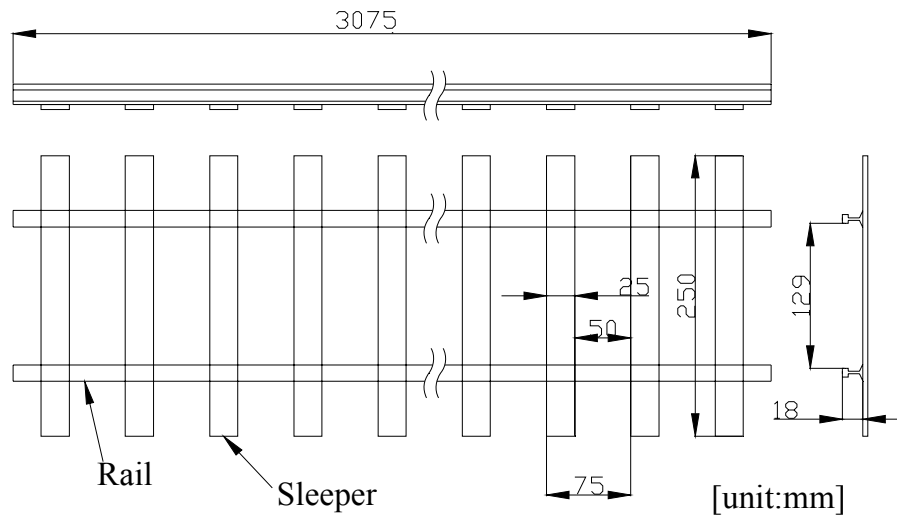

Fig. 3 Track panel 


\subsection{Model trains}

Fig. 4 shows the model train. The scale of the model train was adjusted to $1 / 8.4$ to match the scale of the rails. When we adopt the distance from the underside of the car to the ground as the representative length and the maximum model train speed or the maximum train speed as the representative velocity, the Reynolds number of the model running tests $\left(\operatorname{Re}=5 \times 10^{4}\right)$ is less than that of the on-track tests $\left(\operatorname{Re}=1 \times 10^{6}\right)$ by a factor of $10^{2}$. The blockage ratio of the frontal projected area of the model train to the cross sectional area of the Towing Wind Tunnel Facility is approximately $1 \%$. In the previous wind tunnel tests ${ }^{5)}$, we often used three cars, consisting of head, intermediate and tail cars, by limiting the measurement section length. However, in the model running facility we can use up to five cars, the model train shown in Fig. 4 consists of five cars - three intermediate cars, in addition to the head and the tail cars.

The car is composed of a car body, bogies, and under-floor equipment. The model bogies to match the rails are available on the market. We purchased these bogies and improved their shape as much as possible by attaching bogie frames, a dummy motor, brake disks, and others. We installed the under-floor equipment on the underside of the cars with bolts, to allow altering it easily. We can attach parts for body mount and bogie skirts to the trapezoid under-floor equipment as shown in Fig. 4. The cars have a sufficient inner space to install the measuring devices and cables.

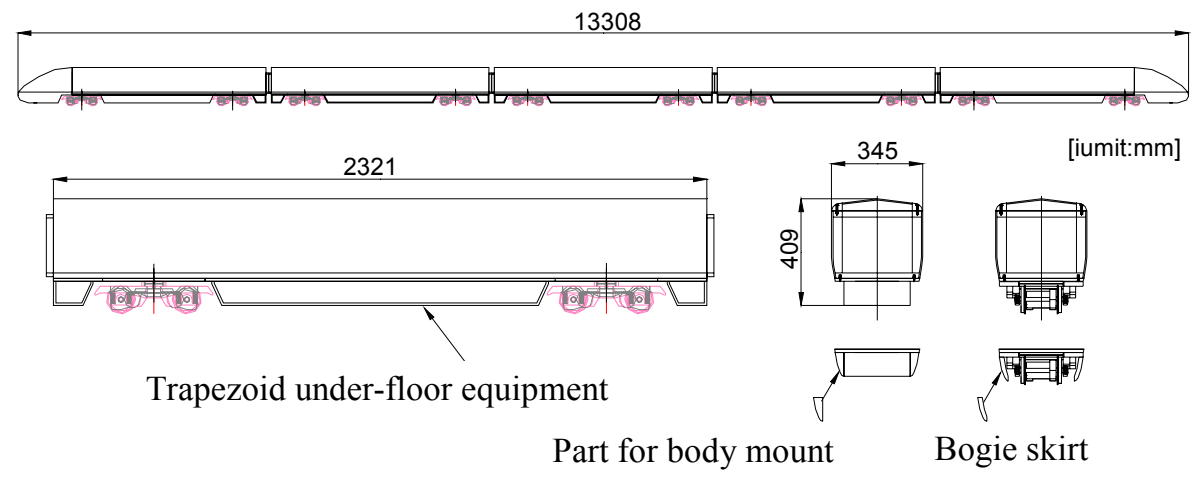

Fig. 4 Model trains

\subsection{Model train traction method}

The model train runs by pulling by a cart Fig. 5 shows the arrangement of the model running facility. The pulling devices consist of an automobile and two carts. Each cart has tires to run and side tires to guide. The head cart is made of pipes and straddles the model train - it is termed a gate car, and it pulls the model train. The next cart is termed a flat cart. A pole connects the flat cart and the automobile. As the blockage ratio of the automobile to the cross section of the Towing Wind Tunnel Facility is the largest of the three devices, running the automobile might influence the upstream flow. Therefore, we set up the flat cart and the connecting pole between the model train and the automobile. This configuration restrains the influence of the automobile on the flow around the model train by keeping sufficient distance between the model train and the automobile. The flat cart is capable of also holding the measuring devices, a battery, a video camera, and other devices.

To run the model train stably, it is important to pull the front edge of the model train of running direction. In addition, it is necessary to suppress vibration from the gate cart, which runs on the asphalt, using tires and guide tires. Therefore, we connected the front and rear edges of the model train to the carts with wire ropes. The wire rope from the front of the 
model train connected to a pole on the front edge of the gate cart and the wire rope from the rear of the model train was connected to the front edge of the flat cart.

The gate cart pushed by the automobile runs and pulls the model train with the wire rope. When the automobile stopped, the model train is pulled by the wire rope from the flat cart and stops. By using an automobile for propulsion, we could run the model train without complex, large, and expensive apparatus. The model train runs smoothly on the rail because it runs and stops depending on the wires, which are free from vibration from the carts.

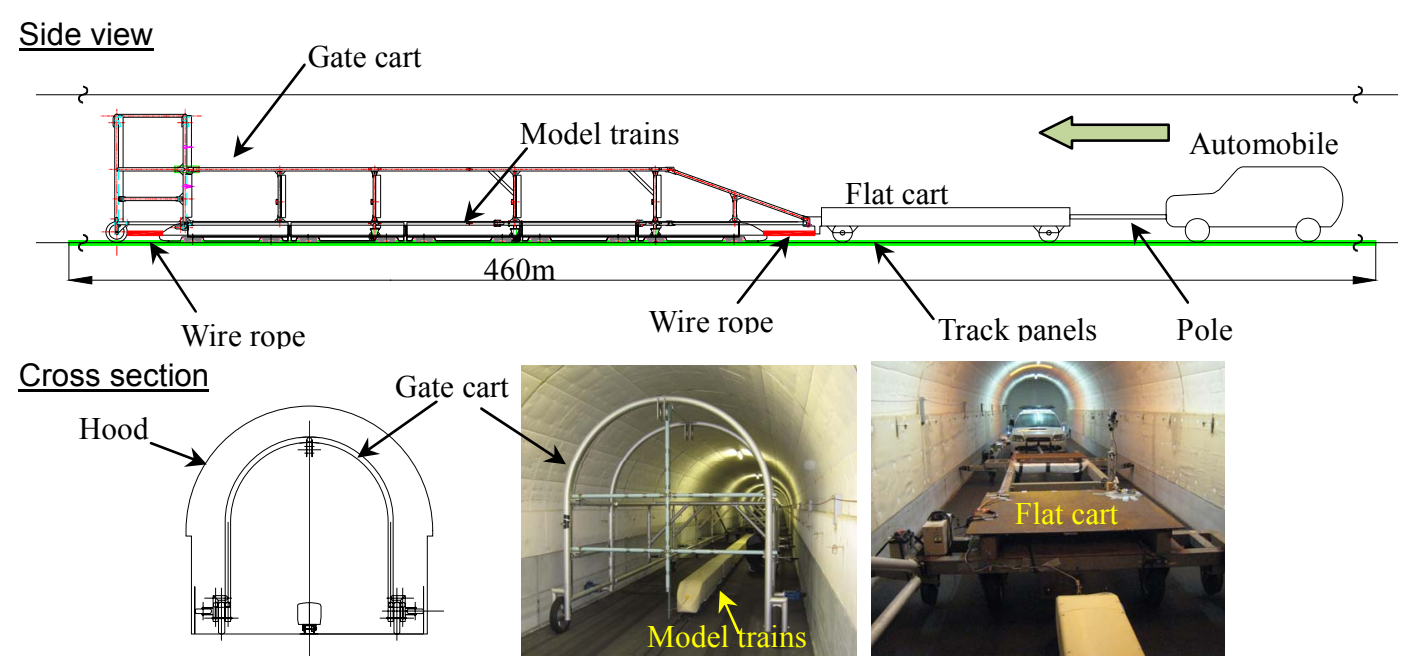

Fig. 5 Arrangement of the model running facility

\subsection{Operation of the model running facility}

During the test, two operators boarded on the automobile. One person drives and the other operates the measuring devices and watches a monitor that projects a live picture of the model train taken by a video camera installed on the flat cart. We prepared a measurement booth (Fig. 2) outside of the test section to operate the measuring devices installed on the ground.

\subsection{Influence of the carts on the flow}

To confirm the influence of the carts on the flow, we measured the flow induced by the carts. We ran the model running facility without the track panels or the model train and measured the flow on the ground, using a hot wire anemometer (ANEMOMASTER MODEL 6141 made by KANOMAX). The measurement point was $10 \mathrm{~mm}$ high, in the center of the cross section, and centered in the direction of the rails. We converted the analogue signal from the anemometer to a digital signal and recorded it in a PC. The sampling frequency was $100 \mathrm{~Hz}$.

Fig. 6 shows the time series data of the flow on the ground. We observed that the air scarcely flowed until the carts passes, that is, the carts had little influence on the upstream flow. 


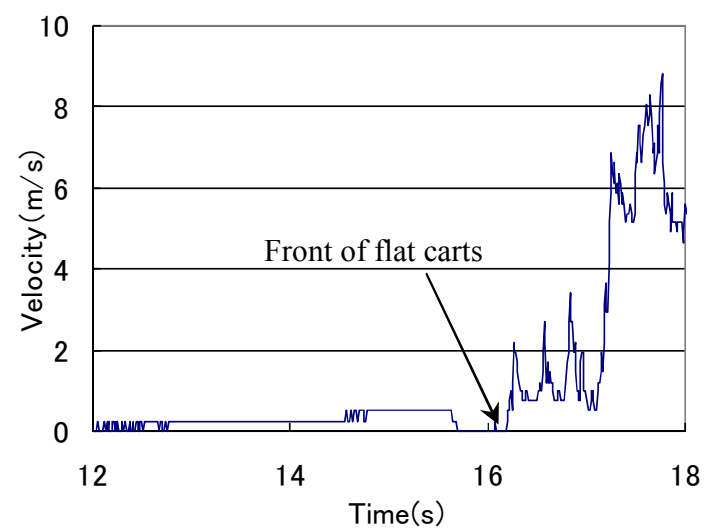

Fig. 6 Time series data of the relative velocity to the ground

\subsection{Measuring the speed of the model train}

For measuring the speed of the model train, we set a laser sensor on the tail bogie and a FV converter (KAZ-7225 made by COCORESEARCH) and data recorder (EZ7510 made by NF Corporation) on the model train.

The output of the sensor was input to the FV converter. The laser sensor counts the rotational frequency of a wheel and it is converted the rotational frequency into an analogue signal by the FV converter, as recorded at a sampling frequency of $100 \mathrm{~Hz}$ by the data recorder.

Fig. 7 shows the run curve of the model train. The curve shows first an acceleration section, next a section at constant speed and last a deceleration section. We performed the measurement of the flow around the model train at the section at a constant speed.

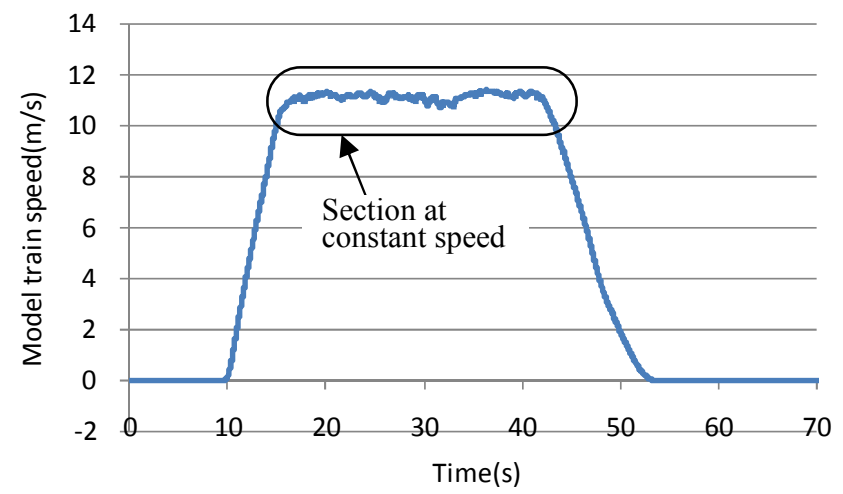

Fig. 7 Run curve of the model train

\section{Flow around the model train}

\subsection{Measurement on the model train}

(1) Method

On one intermediate model car, we installed the comb stagnation pressure tubes in the area under the model train (Fig. 8). In the figure, enlarged drawing shows the measurement points in cross section of the car. We call groups of the measurement points that has same $\mathrm{y}$-coordinate and different z-coordinate "measurement line; W1, W2, W3, W4, W5 and W6". We measured the velocity distribution on the car No.2, No.3 and No.4 by altering the position of the car installed them. We can calculate the velocity from only the stagnation pressure because it seems that the static pressure is equal to the surface pressure of the side 
surface of the car body. The pressure tubes connected the stagnation pressure tubes and the pressure gauge that enabled 48 channel measurement (DSA3217 Digital Sensor Array made by Scanivalve Corporation) on the model train. We connected a reference port of the pressure gauge and the static pressure port on the side surface of the car body with the pressure tubes. LAN cables connected the pressure gauge and the PC on the automobile. We controlled the pressure gauge and measured the pressure in the automobile. The sampling frequency was $125 \mathrm{~Hz}$.

The model train starts from a starting point, accelerates in the first acceleration section of $150 \mathrm{~m}$, runs at a constant test speed in the next test section for $150 \mathrm{~m}$ and decelerates in a deceleration section of $150 \mathrm{~m}$. The speeds of the model train were approximately 10 and 15 $\mathrm{m} / \mathrm{s}$. We controlled its speed by the accelerator in the automobile. We measured accurate airspeed with a pitot tube installed in the upper part of the model train. For the supply of electricity to the measuring devices, we used a battery on the flat cart.
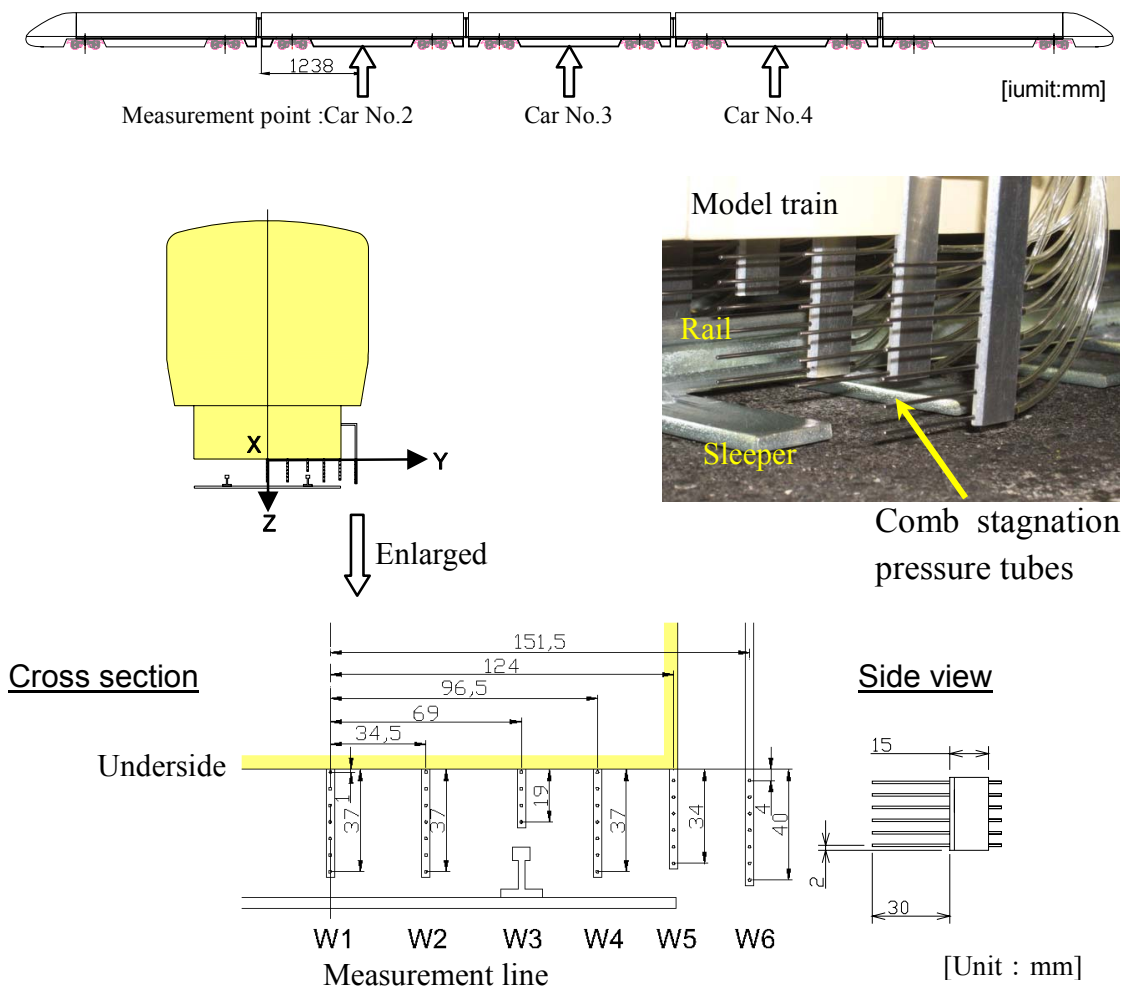

Fig. 8 Comb stagnation pressure tubes

(2) Results and discussion

Fig. 9 shows the velocity distribution at the area under the model train. The test speed of the model train is approximately $15 \mathrm{~m} / \mathrm{s}$. In the figure, the data shows the time average value in the measurement section as a non-dimensional velocity $(u / U)$, made dimensionless by the airspeed measured by the pitot tube. The velocity $(u / U)$ under the car No.3 and the No.4 car is slower than that under the car No.2. The velocity $(u / U)$ distribution becomes almost the same under the car No.3 and the No.4. Therefore, we assume that the velocity distribution at the area under the model train is almost the same as that in the rear cars from the car No.3.

At the centre of the direction of the width of the model train W1, the velocity $(u / U)$ under the car No.3 and the No.4 is approximately 0.5 at the distance of the 0.5 and the velocity distribution is similar to the velocity distribution of the turbulent Couette flow. On 
the other hand, in the measurement line $\mathrm{W} 4$, the velocity $(u / U)$ is larger than 0.5 at the distance of the 0.5 , because the measurement line $\mathrm{W} 4$ is far from the center and the velocity distribution at W4 is affected by the influence of the ground larger than by the model train.

Fig. 10 shows the velocity distribution in the direction of y-axis. The distance means non-dimensional $z$-coordinate by the distance from the underside of the car to the ground $(45 \mathrm{~mm})$. We calculated the velocity distribution at the indicated distance by linear interpolation using the two velocities near the distance. The velocity $(u / U)$ between the rails is less than the velocity $(u / U)$ outside of the rail. The tendency appears more remarkably as the distance from the car body becomes greater.
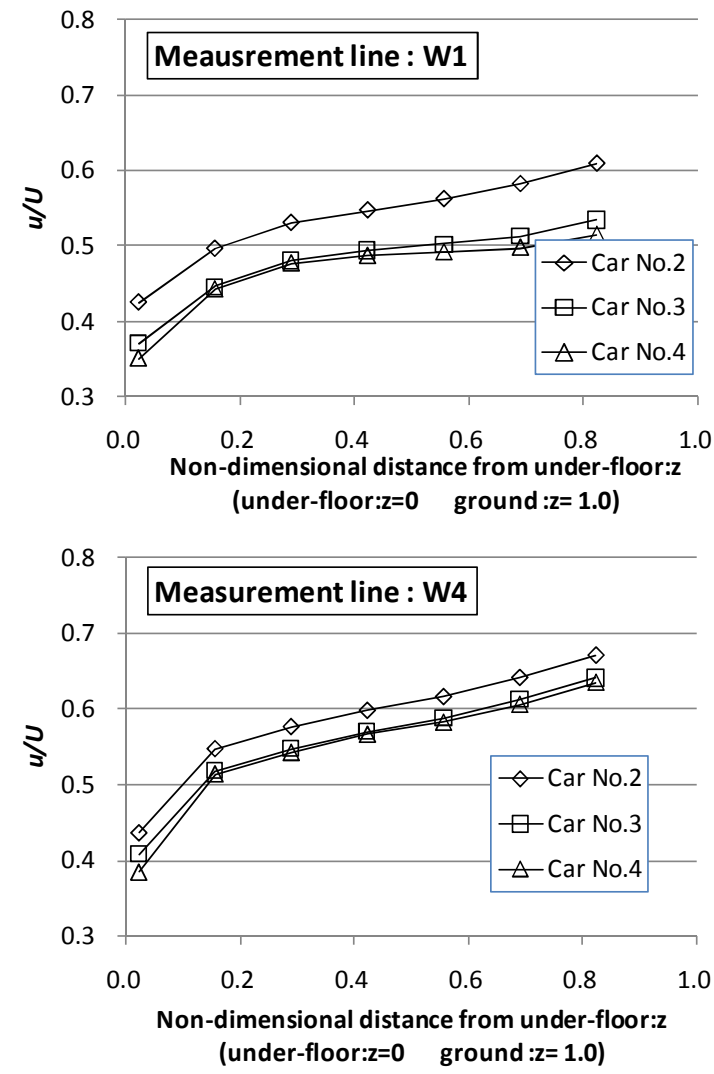

Fig. 9 Relative velocity distributions to the model train (vertical direction) 

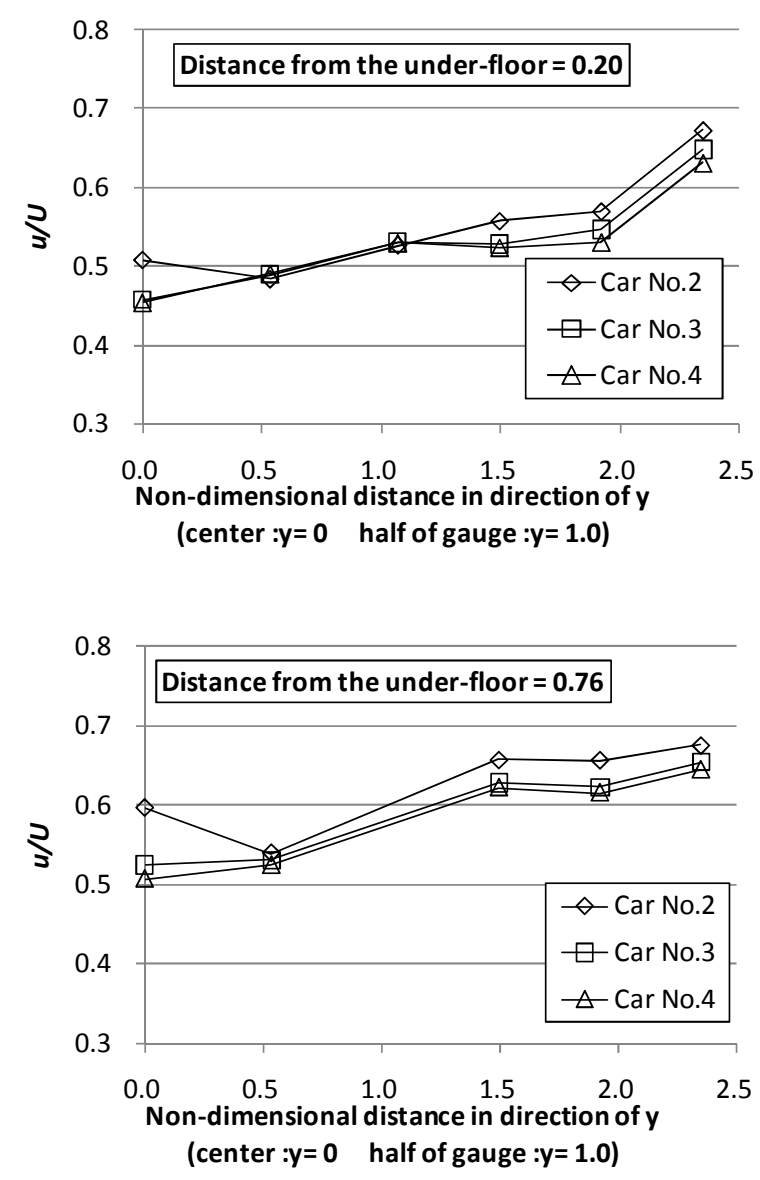

Fig. 10 Relative velocity distributions to the model train (horizontal direction)

\subsection{Measurement on the ground}

(1) Method

We measured the velocity of the flow near the ground by means of a hotwire anemometer (Constant Temperature Anemometry made by DANTEC DYNAMICS). Fig. 11 shows the arrangement the hot wire anemometer sensor installed on the track panel. The sensor installed in the center of the rails and in $22 \mathrm{~mm}$ high. A cable from the sensor leads from the test section to the measurement booth (Fig. 2). We recorded the output of the hotwire anemometer with a PC at a sampling frequency of $1 \mathrm{kHz}$. To detect the model train passing, we set a laser displacement gauge on the sidewall of the test section. The cable of the gauge also led from the test section to the measurement booth. The pipe of the gate cart passing in front of the gauge indicated the passing of the model train. Moreover, we can use this to calculate the speed of the model train, because the gate cart has several pipes, and we know the distance between the pipes. In the tests, the speeds of the model train were approximately $10 \mathrm{~m} / \mathrm{s}$. 


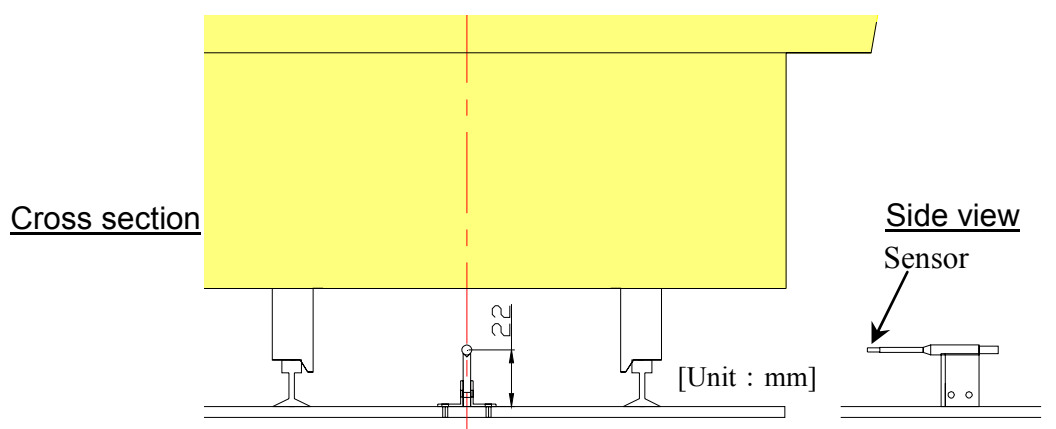

Fig. 11 Hot wire anemometer installed above track panel

(2) Results and discussion

Fig. 12 shows the relative velocity to the ground. We obtained time series data at $10 \mathrm{~m} / \mathrm{s}$, which indicated that the velocity increased at the head, then it maintained almost constant values in the intermediate section, and it temporarily increased further at the tail-this was similar to the data obtained in the on track test ${ }^{7}$.

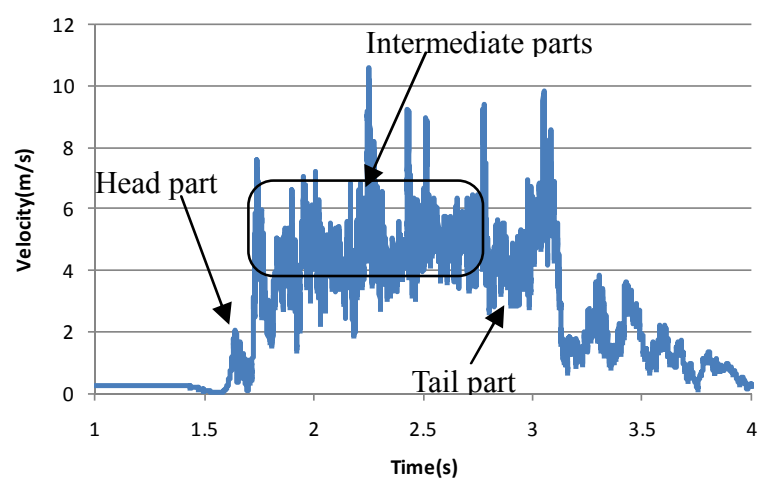

Fig. 12 Time series data of the relative velocity to the ground while train passing

\section{Conclusion}

We developed a model running facility to study the under-floor flow of the car. The facility was set up in the test section of the Towing Wind Tunnel Facility where we removed disturbances, such as natural wind by the hood. By using an automobile for propulsion, we were able to make the model train run without complex, large, and expensive apparatus. The model train runs smoothly on the rail because it is pulled by wire ropes.

On the model train, we measured the velocity distribution around the running model train. The velocity distribution at the area under the model train was almost the same as the velocity distribution in the rear cars from the car No.3, and the velocity between the rails was less than the velocity outside of the rail. Using the facility, we measured the relative velocity to the ground when the model train passed.

\section{References}

(1) Yoshida, M., Uchida, M., Yaguchi, N. and Mifune, N.: Countermeasures for Ballast-Flying Phenomena by High-Speed Trains, RTRI REPORT, Vol. 6, No. 6 (1992), 27-36 (in Japanese).

(2) Kawashima, K., Endo, T. and Fuji, T.: Experimental Study on Prevention of Snow-Flying Phenomena Caused by High-Speed Shinkansen Trains, RTRI REPORT, Vol. 8, No. 7 (1994), 7-12 (in Japanese). 
(3) A. Ido and Y. Kohama: A Study on Reducing Aerodynamic Drag of Trains by Smoothing the Under-floor Surface, Transactions of the Japan society of mechanical engineering, Series (B), Vol. 71, No. 703 (2005), 73-80 (in Japanese).

(4) T. Takaishi, Y. Zenda, A. Sagawa and K. Nagakura: Study on the Aeroacoustic Noise from the Lower Part of the High Speed Train, Transactions of the Japan society of mechanical engineering, Series (B), Vol. 67, No. 662 (2001), 2478-2486, (in Japanese).

(5) IDO, A. et al.: Wind Tunnel Test to reduce aerodynamic drag of trains by smoothing the under-floor construction, Quarterly Report of RTRI, Vol. 42, No. 2 (2001), 94-97.

(6) Yoshioka, S., Kikuchi, S., Ohta, F., Kato, T., Song, J. and Kohama, Y.: Measurement of Ground Effect and Boundary Layer Transition by Towing Wind Tunnel, Fluid Dynamics Research, 41(2009), 021408(12pp).

(7) A.Ido, S.Saitou, K.Nakade, S.Iikura: Study on under-floor flow to reduce ballast flying phenomena, Proceedings of $8^{\text {th }}$ World Congress on Railway Research, CD-ROM (2008). 